\title{
Photo Material Readiness at the Eve of EUVL HVM
}

\author{
Danilo De Simone*, Pieter Vanelderen, and Geert Vandenberghe
}

\author{
IMEC, Kapeldreef 75, 3001 Leuven, Belgium \\ *danilo.desimone@imec.be
}

\begin{abstract}
In the last years, the continuous efforts on the development of extreme ultraviolet (EUV) lithography has allowed to push the lithographic performance of the EUV photoresists on the ASML NXE:3300 full field exposure tool and today both chemically amplified (CAR) and metal-oxide (MOR) EUV photoresists have been introduced for patterning imec's $7 \mathrm{~nm}$ node critical layers. However, the HVM requirement to have a cost-effective high sensitivity photoresist $\left(<20 \mathrm{~mJ} / \mathrm{cm}^{2}\right)$ still remains a big challenge and further efforts are needed to improve the photoresist sensitivity without affecting resolution and patterning quality. In this work, we present the results of the best performing photoresists (both CAR and MOR) at low exposure dose for dense line-space patterns at $32 \mathrm{~nm}$ pitch, dense contact holes at $36 \mathrm{~nm}$ pitch and dense pillars at $38 \mathrm{~nm}$ pitch, reporting the most critical patterning challenges for the investigated structures. Furthermore, we discuss the role of the substrate underneath the EUV photoresist and its impact on the lithographic EUV process setup from both patterning and light-matter interaction standpoint. Finally, we introduce the tone reversal process (TRP) as alternative capability for pillar patterning.
\end{abstract}

Keywords: EUVL, Low exposure dose EUV resist, EUV resist sensitivity, EUV substrate

\section{Introduction}

The shortening wavelength trend of exposure tools is continuing with the aim of achieving sub$20 \mathrm{~nm}$ half-pitch resolution mass production by the deployment of the EUVL at $13.5 \mathrm{~nm}$ wavelength.

Current EUV baseline processes using the ASML NXE3300 full field scanner have been setup for the critical layers of the imec N7 (iN7) BEOL process modules with a resist sensitivity of $35 \mathrm{~mJ} / \mathrm{cm}^{2}$, $40 \mathrm{~mJ} / \mathrm{cm}^{2}$ and $60 \mathrm{~mJ} / \mathrm{cm}^{2}$ for metal, block and vias layer, respectively (Fig. 1). However, EUV lithography requires high-performance photoresists with high sensitivity $\left(<20 \mathrm{~mJ} / \mathrm{cm}^{2}\right)$ to secure high scanner throughput in HVM environment. Therefore imec has started in 2016 a dedicated EUV photoresist screening activity aiming to reach the required exposure dose and assess the lithographic performance of such a class of EUV photoresists. The high sensitivity photoresist screening work is conducted with strong attention to their manufacturability, but also exploratory research for novel solutions is carried out.

Such a study reveals that photoresist formulations with a cost-effective resist sensitivity are feasible today, however the pattern defectivity at nano scale look the major limiting factor of the lithographic process window and further efforts are needed to reach a HVM maturity level.

\begin{tabular}{|c|c|c|}
\hline $\begin{array}{c}\text { Metal } 2 \\
32 \mathrm{~nm} \text { pitch } \\
\mathrm{CD} \text { tg }=16 \mathrm{~nm} \\
\text { Dose }=34.5 \mathrm{~mJ} / \mathrm{cm}^{2} \\
\text { LWR }=4.5 \mathrm{~nm} \\
\end{array}$ & $\begin{array}{c}\text { Block } \\
42 \mathrm{~nm} \text { min pitch } \\
\mathrm{CD} \text { min tg }=21 \times 32 \mathrm{~nm} \\
\text { Dose }=42 \mathrm{~mJ} / \mathrm{cm}^{2} \\
\text { LWR }=3.0 \mathrm{~nm} \\
\end{array}$ & $\begin{array}{c}\text { Vias I } \\
53 \mathrm{~nm} \text { min pitch } \\
\mathrm{CD} \mathrm{tg}=21 \times 32 \mathrm{~nm} \\
\text { Dose }=66 \mathrm{~mJ} / \mathrm{cm}^{2} \\
\mathrm{LWR}=2.9 \mathrm{~nm} \\
\end{array}$ \\
\hline & 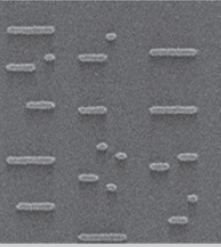 & 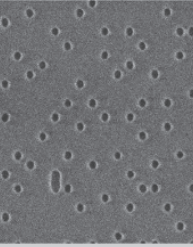 \\
\hline
\end{tabular}

Fig. 1. Lithographic patterning state-of-the-art for imec BEOL $7 \mathrm{~nm}$ node. Three applications with different feature types and sizes are reported: line space by CAR (metal layer for single EUV exposure), block by MOR (metal cut for SAQP metal patterning), holes by CAR (vias patterning).

As a consequence of targeting the highest productivity with low exposure dose, fundamental studies on light-matter interactions have become essential to develop a high performing EUV 
photoresist. In this context, different substrates typically used underneath an EUV photoresist, have been examined looking at the photoemission response when exposed with a light at $91.6 \mathrm{eV}$ (photon energy at $13.5 \mathrm{~nm}$ ). A correlation with the patterning performance indicates that substrates with higher photoemission response have smaller depth-of-focus (DoF). Furthermore, the exposure dose of the photoresist and the related line-withroughness (LWR) and local-critical-dimension uniformity (LCDU) can be modulated by changing the chemistry design of the substrate confirming the important role of the substrate in the overall resolution (R), line-with-roughness (L), sensitivity (S) EUV photoresist performance.

Further, because the current state-of-the-art for the EUV mask fabrication is the dark tonality as favored path to reduce the defectivity concerns on the mask blank, a patterning exploration in single EUV print on dense pillar patterning has been conducted looking at negative tone photoresist systems [1]. Furthermore, a feasibility study to make pillar patterning on wafer has been carried out by applying a tone reversal process (TRP) on a high resolution positive tone system for dense staggered contact hole process at $40 \mathrm{~nm}$ and $38 \mathrm{~nm}$ pitches. The initial experiments are promising and they indicate the TRP as a viable and alternative post litho process path to the single EUV print to make dense pillar pattern on wafer.

The experimental setup and the results of the low dose exposure dose EUV photoresist screening activity at imec, the EUV substrate investigation and the positive tone reversal process are described and discussed in the next paragraphs.

\section{Experimental}

\subsection{Patterning}

All the photoresists were developed and provided by different photoresist suppliers. A $300 \mathrm{~mm}$ TEL CLEAN TRACK ${ }^{\mathrm{TM}}$ LITHIUS Pro ${ }^{\mathrm{TM}}$ Z-EUV tool and ASML NXE3300B full field EUV scanner tool with customized illuminations were used for litho patterning. All the photoresist samples were coated on stack organic HM. 65nm film thickness was used for the spin-on-carbon (SOC) and $10 \mathrm{~nm}$ film thickness for the spin-on-glass (SOG). Top-down images, CD, LWR, LCDU measurements were done on the HITACHI CG5000 scanning electron microscope.

2.2. Photo electron emission of photo material at EUV wavelength

Photo drain current measurements were performed by X-Ray Absorption Spectroscopy (XAS) at the BEAR beamline at Elettra, Synchrotron facility in Italy. Photocurrent measurements were performed on photo materials coated on bare silicon chips irradiated with a spot light at $91.6 \mathrm{eV}$ energy [2]. The photocurrent was measured from the ground insulated sample inside the vacuum chamber and connected through an electrical vacuum feedthrough to a pico-ampere meter. The measurements were performed at room temperature in the BEAR spectroscopy chamber. The data were normalized to the impinging photon intensity (I0), measured by removing the sample and subsequently placing a calibrated detector. All the photo drain current measurements were finally treated to calculate the photon electron emission (Yph-e) of each analyzed photo material sample.

\section{Results and discussion}

3.1. Low exposure dose EUV line-space (LS) resist screening

The goal of the low exposure dose LS EUV photoresist activity was to identify photoresist candidates with high sensitivity that can satisfy manufacturability requirements. Therefore the linespace pitch target and exposure illumination mode were selected in line with the metal 2 layer pitch of the imec BEOL $7 \mathrm{~nm}$ node that it is equal to $32 \mathrm{~nm}$. The substrate underneath the resist was given to the material suppliers as additional variable of the process setup with the only constraint of being etchable and thus to enable the pattern transfer in following process. CD and LWR targets after development (ADI) were set equal to $16 \mathrm{~nm}$ and < $3.2 \mathrm{~nm}$, respectively. A summary of the key patterning requirements are reported in the Table 1 .

Table 1. Requirements for $16 \mathrm{~nm}$ dense line-space pattern high sensitivity EUV photoresists screening on imec NXE3300 scanner tool.

\begin{tabular}{ll}
\multicolumn{1}{c}{$\begin{array}{c}\text { 32nm Pitch } \\
\text { I6nm dense LS }\end{array}$} & \multicolumn{1}{c}{ Requirements } \\
\hline Dose-to-Size target & $20 \mathrm{~mJ} / \mathrm{cm}^{2}$ \\
LWR & $<3.2 \mathrm{~nm}$ \\
Illumination & customized \\
Mask tonality & Dark \\
Resist Film Thickness & I8-30nm range \\
\hline
\end{tabular}

Five photoresist suppliers were involved and 310 wafers were exposed on the imec EUV cluster in eight months of 2016. The count of wafers includes different photoresist samples, process 
conditions and substrates. All the tested CARs were positive tone imaging systems. Only the MOR was a negative tone imaging system. Top-down CDSEM images from the best photoresist sample per supplier of dense lines with a pitch of $32 \mathrm{~nm}$ are reported in Fig. 2 at the best exposure conditions.

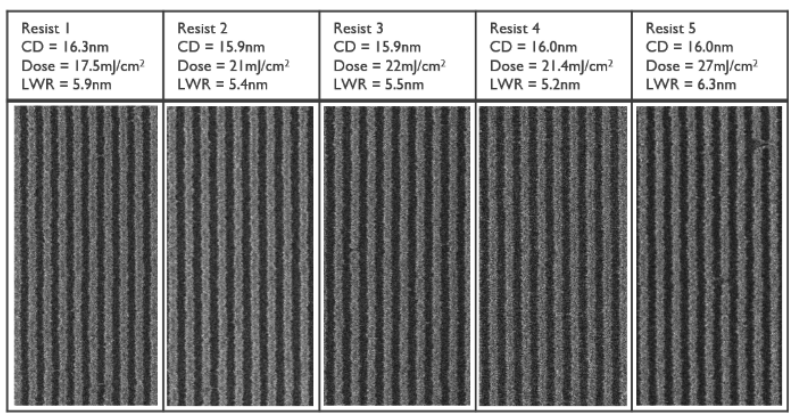

Fig. 2. Top-down CD-SEM images. Obtained CD and LWR of $16 \mathrm{~nm}$ dense line pattern in rectangular scan mode. Each image is from the best sample per photoresist supplier at the best exposure conditions.

Results clearly indicate that all the suppliers were able to enter in the low exposure dose regime, however the LWR turned out as the most difficult target to achieve showing a range value of 5.2-6.3 $\mathrm{nm}$, significantly above the target of $<3.2 \mathrm{~nm}$. Furthermore, a fundamental defectivity issue was encountered in all the examined photoresists. The presence of nano-bridges and broken lines was observed as primary source of defectivity for chemically amplified resists whereas only nanobridges were observed for metal-oxide photoresist. A representation of the defect type for each resist type is reported in Fig. 3.

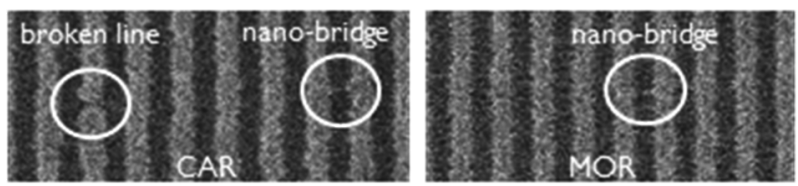

Fig. 3. Top-down CD-SEM images of $16 \mathrm{~nm}$ dense linespace pattern in rectangular scan mode and representative defectivity observed on low exposure dose resist sample. on the left: CAR, on the right: MOR.

\subsection{Low exposure dose contact hole $(\mathrm{CH})$ EUV resist screening}

The goal of the low exposure dose activity (currently running at imec) for EUV contact hole photoresist is to initially explore the feasibility of a high sensitivity photoresist while pushing the resolution capability. For such a reason $36 \mathrm{~nm}$ pitch target for dense contacts was selected. As the activity is at the early stage an organic underlayer was selected as substrate underneath the photoresist.
CD and LCDU targets after development (ADI) were set equal to $18 \mathrm{~nm}$ and $<3.0 \mathrm{~nm}$, respectively. A summary of the key patterning requirements are reported in Table 2.

Table 2. Requirements for dense $18 \mathrm{~nm}$ contact hole pattern high sensitivity EUV photoresists screening on imec NXE3300 scanner tool

\begin{tabular}{ll}
\hline \multicolumn{1}{c}{$\begin{array}{c}\text { 36nm Pitch } \\
\text { I8nm dense CH }\end{array}$} & \multicolumn{1}{c}{ Requirements } \\
\hline Dose-to-Size target & $30 \mathrm{~mJ} / \mathrm{cm}^{2}$ \\
LCDU & $<3.0 \mathrm{~nm}$ \\
Illumination & customized \\
Mask tonality & Dark \\
Resist Film Thickness & $50 \mathrm{~nm}$ \\
\hline
\end{tabular}

Four photoresist suppliers were involved in the first round of 2017 of such a photoresist screening activity. 152 positive tone CAR samples and 238 wafers were exposed on the imec EUV cluster in two months. The count of wafers includes different photoresist samples and process conditions. Topdown CD-SEM images from the best photoresist sample per supplier of dense and regular contact holes with a pitch of 36nm are reported in Fig. 4 at the best exposure conditions.

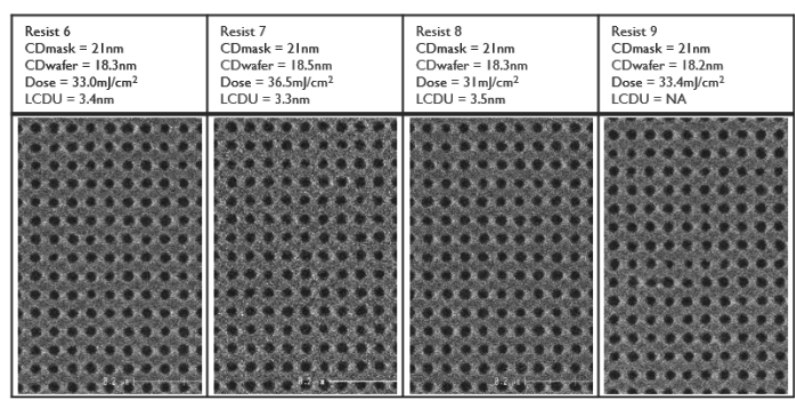

Fig. 4. Top-down CD-SEM images. Obtained CD and LCDU of $18 \mathrm{~nm}$ dense and regular contact hole pattern in square scan mode. Each image is from the best sample per photoresist supplier at the best exposure conditions.
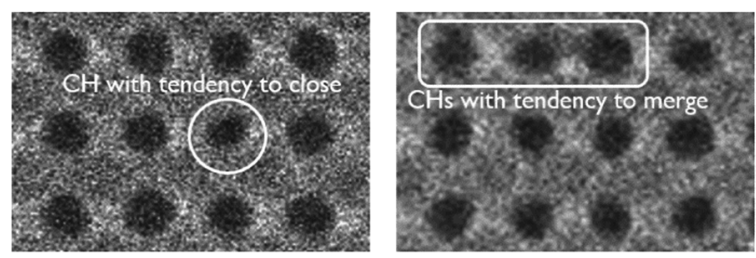

Fig. 5. Top-down CD-SEM images of $18 \mathrm{~nm}$ dense contact hole pattern square scan mode and representative pattern defectivity observed on low exposure dose resist samples.

Results clearly indicate that all the suppliers were able to enter in the low exposure dose regime 
with a LCDU value close to the target, however a fundamental defectivity issue as tendency to have randomized closed or merged contact holes was observed in all the examined photoresist as primary source of defectivity. A representation of the defect type is reported in Fig. 5.

\subsection{EUV substrates}

The impact of the lithographic performance of different substrates underneath the photoresist has been explored by looking at contact hole and linespace EUV patterning. In the first case a pitch equal to $44 \mathrm{~nm}$ were exposed by using the same photoresist on three different substrates (Fig. 6).

\begin{tabular}{|c|c|c|c|}
\hline & $\begin{array}{c}\text { Organic } \\
\text { underlayer (UL) }\end{array}$ & $\begin{array}{l}\text { Metal Hard Mask } \\
\text { (MHM) }\end{array}$ & $\begin{array}{l}\text { Spin-on-Glass } \\
\text { (SOG) }\end{array}$ \\
\hline $\begin{array}{l}\text { Pitch } 44 \mathrm{~nm} \\
\text { CDmask: } 26 \mathrm{~nm} \\
\text { CDtg: } 24 \mathrm{~nm} \\
\text { (on wafer) }\end{array}$ & 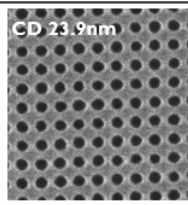 & 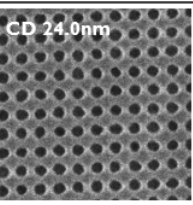 & 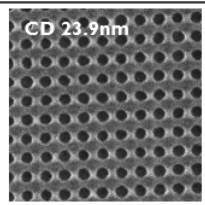 \\
\hline Dose $\mathrm{m} / / \mathrm{cm}^{2}$ & 37 & 20.5 & 37 \\
\hline
\end{tabular}

Fig. 6. Top-down CD-SEM images of dense and regular contact hole pattern $44 \mathrm{~nm}$ pitch on three different substrate types. The MHM substrate delivers 44\% exposure dose less w.r.t. the exposure dose obtained on UL and SOG to get the same CD.

The nature of these three substrates were quite different having as reference an organic underlayer (UL) and as second and third substrate a metal hardmask (MHM) and a spin-on-glass silicon containing hard mask (SOG), respectively. All the three different processes were able to deliver $24 \mathrm{~nm}$ dense contact holes on wafer, however the exposure dose obtained on MHM substrate was 44\% less the exposure dose found the exposure on UL and SOG indicating a possible and significant role of the metal species present in the MHM.

Further experiments on photon-electron emission performed at $91.6 \mathrm{eV}$ on the same three substrates used for the contact hole patterning test above described have shown a different Yph-e behavior. A correlation between Yph-e and the depth-of-focus (DoF) of the respective contact hole process were observed in analogy with the recent observation obtained on $16 \mathrm{~nm}$ line-space patterning [3]. The experiments indicate that the substrate that delivers the lowest Yph-e provides the largest DoF as reported in the chart of Fig. 7.

Further experiments by looking at $16 \mathrm{~nm}$ linespace patterning were carried out with the intention of exploring a specific design property of the substrate and its eventual correlation with the exposure dose of the photoresist. The initial results reported in Fig. 8 show that the exposure dose of the investigated photoresist can be modulated by $15 \%$ by changing the selected property of the substrate. Further investigations are needed for assess the impact on LWR.

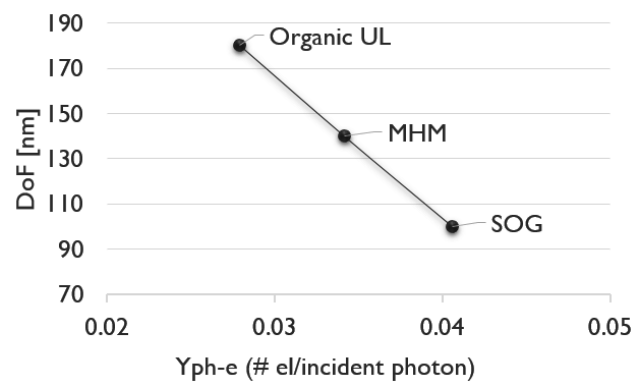

Fig. 7. Depth-of-Focus (DoF) of $44 \mathrm{~nm}$ pitch dense contact hole patterning on three different substrates (UL, MHM, SOG) vs. photon-electron emission of the related substrate (UL, MHM, SOG) used in the patterning experiment. The same photoresist is used in all three patterning experiments. The DoF seems to correlate with the Yph-e.

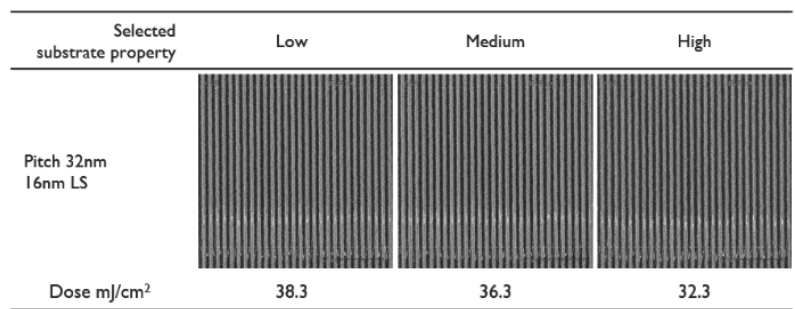

Fig. 8. Modulation of the exposure dose for $16 \mathrm{~nm}$ dense line-space pattern by changing (low, medium, high) a specific property of the substrate underneath the photoresist. $15 \%$ exposure dose variation range is seen in two extreme studied cases (low and high).

\subsection{Pillar (PL) patterning in EUVL}

As part of the exploratory work, pillar pattering capability for tight pitches was checked across all the photoresist suppliers. Due to the constrain of the EUV mask fabrication that requires a dark tonality [1], only few negative tone photoresist systems were available for testing to make pillars on wafer in a single EUV print. The metal-oxide photoresist was the only material able to deliver $38 \mathrm{~nm}$ dense pillar patterning with the limitation of reaching a larger ultimate printable CD of $20.5 \mathrm{~nm}$ (instead of $19 \mathrm{~nm}$ ) with given illumination setting. Top-down SEM images of dense pillar patterning by MOR and the used illuminator shape are reported in Fig. 9.

A feasibility study to make pillar patterning on wafer from a positive tone CAR system has been carried out by applying a tone reversal process (TRP) on two configurations of dense staggered contact hole patterning with minimum contact-to- 
contact distance of $40.3 \mathrm{~nm}$ and $38.3 \mathrm{~nm}$, respectively (Fig. 10). As tone reversal process the dry development rinse material (DDRM) [4] was selected and applied to a low exposure dose contact hole photoresist after the development step. The results reported in figure 10 show the conversion of holes to pillars by DDRM after DDRM etch back and resist stripping. A comparable or slight worse pillar LCDU (4.09 nm and $4.89 \mathrm{~nm}$ ) with respect the original contact hole LCDU (3.93 nm and $3.96 \mathrm{~nm}$ ) for $40 \mathrm{~nm}$ and $38 \mathrm{~nm}$ pitch was obtained, respectively. A CD bias on average of $+3 \mathrm{~nm}$ was obtained converting $20 \mathrm{~nm}$ contact holes to pillars. The TRP process is at the early development stage and large margin on process optimization is possible to push down the LCDU and reach the target of 3 nm.

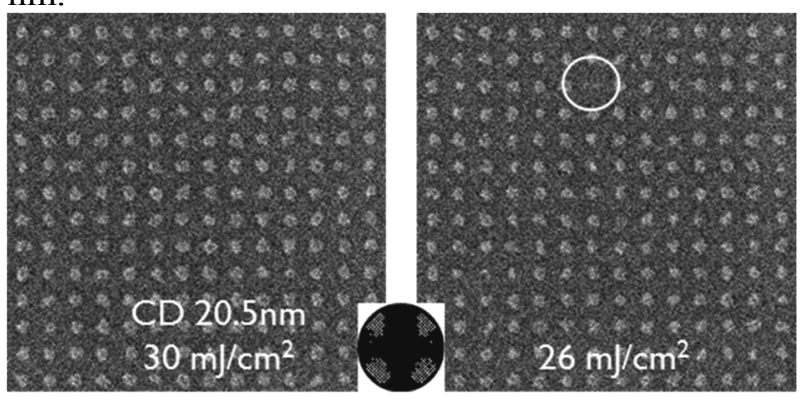

Fig. 9. Top-down CD-SEM images of dense and regular pillar pattern $38 \mathrm{~nm}$ pitch by metal-oxide photoresist. On the left: the ultimate CD of $20.5 \mathrm{~nm}$ delivered with a LCDU of $3.9 \mathrm{~nm}$. On the right: missing pillars are observed as CD goes below $20.5 \mathrm{~nm}$. In the center: the shape of the used illuminator is shown.
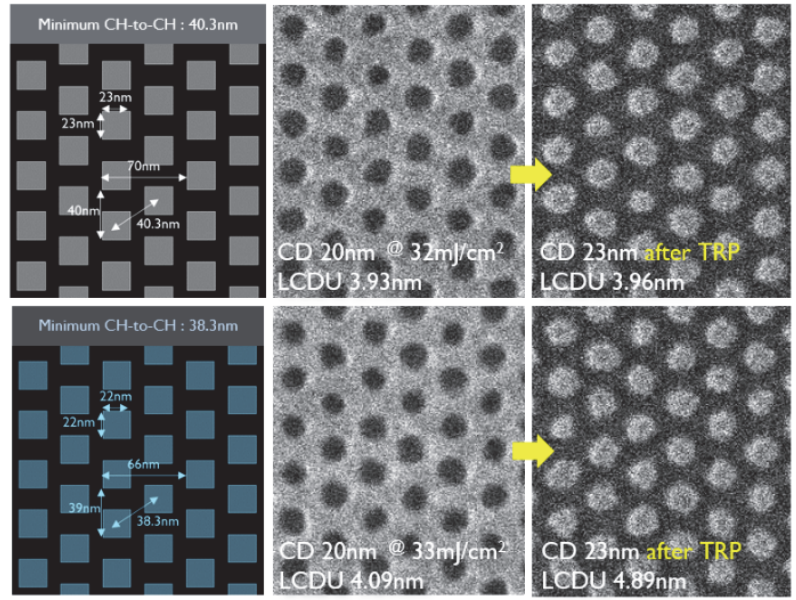

Fig. 10. Staggered pillar patterning with tone reversal process (TRP) by dry development rinse material (DDRM). Two studied cases with minimum contact-tocontact distance $40.3 \mathrm{~nm}$ (top row) and $38.3 \mathrm{~nm}$ (bottom row). On the left: staggered contact hole layout. In the center: contact hole patterning with positive tone CAR system. On the right: pillar patterning by TPR and DDRM from initial positive tone contact hole EUV patterning.

\section{Conclusion}

Extreme-ultraviolet lithography is at the eve of HVM and it requires high-performance photoresists with high sensitivity $\left(<20 \mathrm{~mJ} / \mathrm{cm}^{2}\right)$ to secure high scanner throughput.

EUV photoresist formulations for tight pitches as $32 \mathrm{~nm}$ for dense line-space and $36 \mathrm{~nm}$ for dense contact holes, with a cost-effective resist sensitivity are feasible today, however the pattern defectivity at nano-scale currently seem to be the major limiting factor of the lithographic process window and further efforts are needed to reach a HVM maturity level.

Besides the photoresist, the substrate underneath can play a role in the overall lithographic performance and further fundamental investigations are needed in this respect to identify the right chemical design and quantify pros and cons on the RLS performance of the photoresist.

Pillar patterning limitations when pushing the resolution are today seen in single EUV print with the current photoresists available in the market. Tone reversal process (TRP) from contacts to pillars can be considered as an alternative and valid to enable dense pillar patterning with pitch equal or below $40 \mathrm{~nm}$.

\section{Acknowledgements}

The authors would like to thank the material suppliers at imec (FFEM, JSR, ShinEtsu, TOK, Inpria, Brewer Science and Nissan Chemical) for their participation in the EUV photoresist screening and pattern exploration activities. Furthermore, the authors would like to explicitly thank TOK and Nissan Chemical for supporting the exploration of the tone reversal process.

\section{References}

1. D. De Simone, M. Ming, M. Kocsis, P. De Schepper, F. Lazzarino, G. Vandenberghe, J. Stowers, S. Meyers, B. L. Clark, A. Grenville, V. Luong, F. Yamashita, and D. Parnell, Proc. SPIE, 9776 (2016) 97760B.

2. D. De Simone, M. Ming, F. Lazzarino, and G. Vandenberghe, J. Photopolym. Sci. Technol., 29 (2016) 501.

3. D. De Simone, Y. Vesters, A. Shehzad, G. Vandenberghe, P. Foubert, C. Beral, D. Van Den Heuvel, M. Mao, and F. Lazzarino, Proc. SPIE, 10143 (2017) 101430R1.

4. W. Shibayama, S. Shigaki, S. Takeda, R. Onishi, M. Nakajima, and R. Sakamoto, Proc. SPIE, 9776 (2016) 977609. 